\title{
QUADRATIC EIGENVALUE PROBLEMS
}

\author{
A.S. JONES
}

\begin{abstract}
A class of problems in forced convection give rise to quadratic eigenvalue problems. In this paper it is shown that the eigenvalues are necessarily real. The extension of this result to a wider class of problems is also discussed.
\end{abstract}

\section{INTRODUCTION}

The problem of forced convection in a tube of uniform but arbitrary cross-section $D$ gives rise to the differential equation

$$
\nabla^{2} T=k \rho \frac{\partial T}{\partial x}
$$

where $x$ is the co-ordinate in the direction of the flow, and the velocity $\rho$ is a function of the transverse co-ordinates only.

If we now take the two-sided Laplace transform of this equation with respect to the variable $x$, (or equivalently assume exponential behaviour of the solution with respect to $x$ ) we get the eigenvalue problem

$$
\nabla_{1}^{2} \theta+\left(p^{2}-p k \rho\right) \theta=0
$$

for the transform variable $\theta$, where $\nabla_{1}^{2}$ represents the Laplacian operator restricted to the transverse variables, and the boundary conditions on $\partial D$ are either $\theta=0$ or $\frac{\partial \theta}{\partial n}=0$ depending on the boundary conditions attached to the original problem.

The first papers in this field (Shingh [7], Jones [3]) assumed that the eigenvalues were real, a defect that was remedied in particular cases by Davis [1], and by Deavours [2] and Papoutsakis et al [6], who used the techniques introduced by Langer [4]. In this note it is shown that the eigenvalues are real for arbitrary $D$, provided that $\partial D$ is smooth enough to allow Green's formula to be used.

Received 10 June 1987

Copyright Clearance Centre, Inc. Serial-fee code: 0004-9729/88\$A2.00+0.00. 


\section{ANALysis}

For arbitrary twice-differentiable functions $u, v$ on $D$, we have the Green's formula

$$
\iint_{D}\left(v \nabla_{1}^{2} u-u \nabla_{1}^{2} v\right) d S=\int_{\partial D}\left(u \frac{\partial v}{\partial n}-v \frac{\partial u}{\partial n}\right) d S .
$$

If $u$ and $v$ are eigenfunctions of (1), corresponding to $p_{n}$ and $p_{m}$, then either $u$ and $v$ or $\frac{\partial u}{\partial n}$ and $\frac{\partial v}{\partial n}$ vanish on $\partial D$, so that the right hand side is zero, and we have

$$
\iint_{D} u\left(p_{m}^{2}-p_{m} k_{\rho}\right) v d S=\iint_{D} v\left(p_{n}^{2}-p_{n} k_{\rho}\right) v d S
$$

If we now assume that $p_{n}=a+i b, p_{m}=a-i b, u=U+i V$ and $v=U-i V$, we obtain

$$
2 i b \iint_{D}(2 a-k \rho)\left(U^{2}+V^{2}\right) d S=0
$$

so that if $b \neq 0$,

$$
2 a \iint_{D}\left(U^{2}+V^{2}\right) d S=\iint_{D} k \rho\left(U^{2}+V^{2}\right) d S .
$$

The functions $U, V$ satisfy

$$
\begin{aligned}
& \nabla_{1}^{2} U+\left(a^{2}-b^{2}-a k \rho\right) U-(2 a b-b k \rho) V=0 \\
& \nabla_{1}^{2} V+\left(a^{2}-b^{2}-a k \rho\right) V+(2 a b-b k \rho) U=0 \quad \text { on } D,
\end{aligned}
$$

with either $U=V=0$ or $U_{n}=V_{n}=0$ on $\partial D$.

If we now apply the Green's identity

$$
\iint_{D}\left(\underset{\sim}{\nabla} \underset{\sim}{\nabla} v+u \nabla^{2} v\right) d S+\int_{\partial D} u \frac{\partial v}{\partial n} d S=0
$$

we have

$$
\iint_{D}\left(\left(\underset{\sim_{1}}{\nabla} u\right)^{2}+u \nabla_{1}^{2} u\right) d S=0
$$

and

$$
\iint_{D}\left((\underset{\sim 1}{\nabla} v)^{2}+v \nabla_{1}^{2} v\right) d S=0
$$

Substituting for $\nabla_{1}^{2} U$ and $\nabla_{1}^{2} V$ and adding, we obtain

$$
\iint_{D}\left(\left(\nabla_{1} u\right)^{2}+\left(\nabla_{1} v\right)^{2}\right) d S=\iint_{D}\left(a^{2}-b^{2}-a k \rho\right)\left(u^{2}+v^{2}\right) d . S
$$

and, on substituting for $\iint_{D} k \rho\left(U^{2}+V^{2}\right) d S$ from (2)

$$
\iint_{D}\left(\left(\nabla_{1} u\right)^{2}+\left(\nabla_{1} v\right)^{2}\right) d S=-\left(a^{2}+b^{2}\right) \iint_{D}\left(u^{2}+v^{2}\right) d S .
$$

This provides the necessary contradiction to show that there are no complex eigenvalues. 


\section{Discussion}

The exact form of the domain $D$ does not enter into the above analysis, so that the results are applicable not only to simple tubes but also to multiply connected domains, and, with the addition of the appropriate radiation conditions, to external flow problems also. It is also apparent that the boundary conditions can be a mixture of Dirichlet and Neumann types without affecting the validity of the analysis, so that it applies to almost all problems in uni-directional forced convection.

The analysis also extends to the case of diffusion with chemical reaction considered by Lauwerier [5], for which the governing equations are of the form

$$
\nabla^{2} T=k \rho \frac{\partial T}{\partial x}+c T
$$

In this case equation (3) becomes

$$
\iint_{D}\left(\left(\nabla_{1} u\right)^{2}+\left(\nabla_{1} v\right)^{2}\right) d S=-\left(a^{2}+b^{2}+c\right) \iint_{D}\left(u^{2}+v^{2}\right) d S .
$$

However, the constant $c$, which represents the reaction rate, is positive, so that once again the eigenvalues are real.

Finally, it should be noted that while the motivation for this work was forced convection in a uniform tube, which implies a two-dimensional Laplacian operator $\nabla_{1}^{2}$ and a one-signed velocity $\rho$, neither of these restrictions apply to the analysis above, which remains valid irrespective of the dimension of the Laplacian and the behaviour of the function $\rho$.

\section{REFERENCES}

[1] A.M.J. Davis, 'The zeros of a function occurring in the solution of the Graetz problem', Mathematika 21 (1974), 55-63.

[2] C.A. Deavours, 'An exact solution for the temperature distribution in parallel plate poiseuille flow', Trans ASME(C), Journal of Heat Transfer 96 (1974), 489-495.

[3] A.S. Jones, 'Laminar forced convection at Low Péclet number', Bull. Austral. Math. Soc. 6 (1972), 83-94.

[4] R.E. Langer, "The expansion problem in the theory of ordinary linear differential systems of the second order', Trans. Amer. Math. Soc. 31 (1929), 868-906.

[5] H.A. Lauwerier, 'A diffusion problen with chemical reaction', Appl. Sci. Res. A8 (1959), 366-376.

[8] Eleftherios Papoutsakis, Doraiswami Ramkrishna and Henry C. Lim, 'The extended Graetz problem with Dirichlet wall boundary conditions', Appl. Sci. Res. 36 (1980), 13-34.

[6] S.N. Singh, 'The determination of eigen-functions of a certain Sturm-Liouville equation and its application to problems of hear-transfer', Appl. Sci. Res AT (1958), 237-250. 